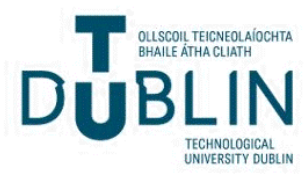

Technological University Dublin

ARROW@TU Dublin

2013-9

\section{Dual Circularly-Polarized Patch Antenna Using Even and Odd Feed-Line Modes}

\author{
Adam Narbudowicz \\ Technological University Dublin, adam.narbudowicz@mydit.ie \\ Xiulong Bao \\ Technological University Dublin, xiulong.bao@tudublin.ie \\ Max Ammann \\ Technological University Dublin, max.ammann@tudublin.ie
}

Follow this and additional works at: https://arrow.tudublin.ie/ahfrcart

Part of the Electromagnetics and Photonics Commons, and the Systems and Communications Commons

\section{Recommended Citation}

Narbudowicz, A., Bao, X. L. \& Ammann, M. J. Dual circularly-polarized patch antenna using even and odd feed-line modes, >i>IEEE Transactions on Antennas \& Propagation, vol. 61, issue 9, pp. 4828-4831, 09/ 2013. doi:10.1109/TAP.2013.2269471

This Article is brought to you for free and open access by the Antenna \& High Frequency Research Centre at ARROW@TU Dublin. It has been accepted for inclusion in Articles by an authorized administrator of ARROW@TU

Dublin. For more information, please contact

arrow.admin@tudublin.ie, aisling.coyne@tudublin.ie, gerard.connolly@tudublin.ie.

Funder: Science Foundation Ireland

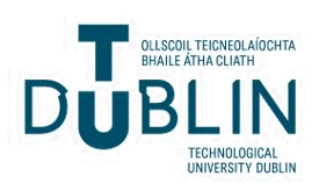




\title{
Dual circularly-polarized patch antenna using even and odd feed-line modes
}

\author{
Adam Narbudowicz, Student Member, IEEE, Xiulong Bao, Senior Member, IEEE, and \\ Max J. Ammann, Senior Member, IEEE
}

\begin{abstract}
This paper proposes a new patch antenna with dual circularly polarized capability at $2.575 \mathrm{GHz}$. The design exploits the even and odd modes in a coplanar waveguide transmission line and enables simultaneous right- and left-handed circular polarization, with two individual excitation ports. A patch antenna is fed by multimode transmission line and the advantages and disadvantages are discussed. A prototyped antenna realized up to $20 \mathrm{~dB}$ isolation between ports with $S_{21}<-$ $10 \mathrm{~dB}$ bandwidth of $87 \mathrm{MHz}$. Realized gains for the two modes of operation are 5.77 and 7.23 dBic. The proposed structure is compact and easy to manufacture.
\end{abstract}

Index Terms-Antenna feeds, circular polarization, dual polarization, microstrip antennas

\section{INTRODUCTION}

$\mathrm{D}$ IVERSITY radio systems offer notably improved quality in wireless communications links. Among the various schemes (space, polarization or frequency diversity) polarization diversity offers good results within limited space and bandwidth. Although it has been applied mainly to linear polarization, recently it was demonstrated that circular polarization (CP) diversity also offers significantly greater channel capacity, especially for line-of-sight propagation [1]. This creates a need for simple and compact dual CP antennas, such as reported in this communication.

There are many reported CP antennas which are electronically adjustable/reconfigurable, implemented by switching a perturbation element [2]-[4], or rerouting current flow on the radiator [5]. Usually either PIN diodes or RFMEMS switches are required, which can increase manufacturing costs. The designs allow the use of either right handed- (RHCP) or left-handed circular polarization (LHCP), but not simultaneously. A good solution for this is to use a dual-fed microstrip patch antenna with a hybrid coupler [6][7]. The coupler ensures the $90^{\circ}$ phase shift needed for $\mathrm{CP}$ and the antenna provides either LHCP or RHCP, depending on the excited port. Due to size limitations, a compact coupler involving varactor diodes [8] is often used.

Manuscript received Nov 19th, 2012. This work was supported by the Science Foundation Ireland under Grant Number 09/SIRG/I1644

A. Narbudowicz, X. L. Bao and M. J. Ammann are with the Antenna \& High Frequency Research Centre, Dublin Institute of Technology, Dublin 8, Ireland (phone: +353-1402-4905; fax: +353-1402-4690; e-mail: max.ammann @ dit.ie).
In this communication we propose a new compact dual CP antenna. The feed exploits the even and odd modes of a coplanar waveguide (CPW) transmission line. Designs based on similar principles have been successfully applied to linear polarization diversity [9], however not for CP and not for microstrip patch antennas. The proposed design enables transmission of two independent signals (for two orthogonal polarizations), which are fed to the patch by the same transmission line, hence providing a high degree of flexibility and space saving for the PCB feed layout, which is desirable in modern compact technologies. Unlike in [9], the use of a microstrip patch antenna is proposed, as slot antennas produce a rearward cross polarized beam, which is not suitable for CP diversity.

\section{FEED TECHNIQUE}

In general each transmission line with three conductors can support two principal modes without a cutoff frequency (these modes are not necessarily TEM or quasi-TEM [10]). For CPW they are often referred to as odd and even, due to the symmetry of their electric field lines. For the even mode there is conceptually a 180 phase shift (electric field vectors directed in opposite directions, as seen in Fig. 1a) between the two slots comprising the transmission line. This corresponds to the most common mode used in CPW, which is very well studied in the literature. For the odd mode there is no phase shift (electric field vectors directed in the same direction, as seen in Fig. 1b) and can be seen as two coupled slotlines (CSL). Similarly to a single slotline, this is a non-TEM mode [10].

Two independent ports were used: port 1 excites the even mode of the CPW (see Fig. 1a), and port 2 excites a microtrip line, which later couples energy into the odd mode of CPW, or CSL (see Fig. 1b). Subsequently, the two slotlines diverge with a zero phase difference for the odd mode and $180^{\circ}$ for the even mode. With an additional $90^{\circ}$ section in one path, the resulting phase shifts between the slots are $270^{\circ}$ and $90^{\circ}$ which, when applied to antenna feeds, results in RHCP and LHCP. 


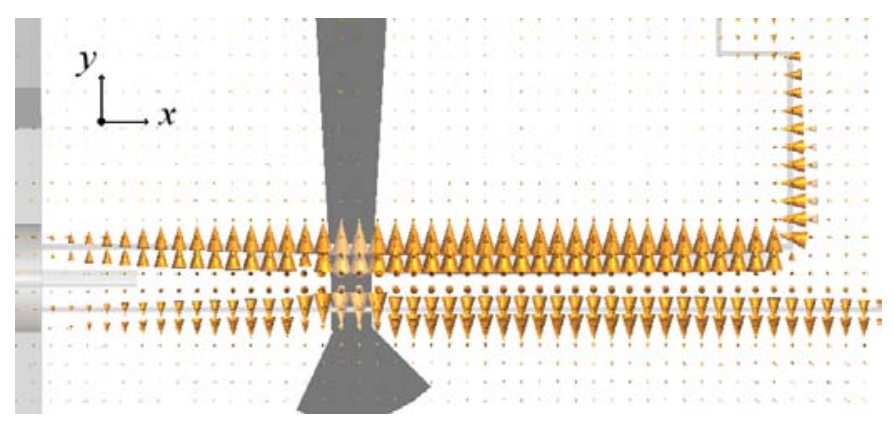

a)

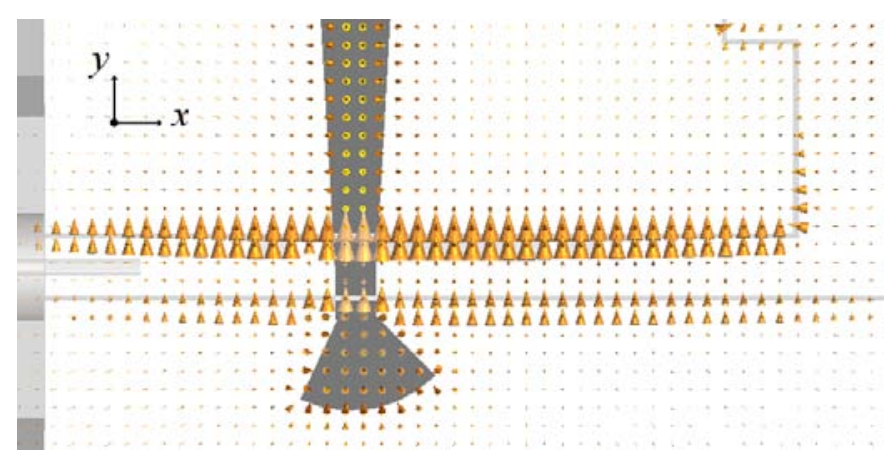

b)

Fig 1. Simulated electric field in the feed line (a) even mode, excited by port 1,(b) odd mode, excited by port 2 (microstrip line).

\section{EXPERIMENTAL VALIDATION}

Fig. 2 shows the proposed design and Fig 3 photos of the prototype antenna. The structure was implemented on a single layer of Taconic RF-35 substrate ( $h=1.5 \mathrm{~mm}, \varepsilon_{r}=3.5$ ) to avoid potential problems with prepreg glue in the feed area. Only the patch is elevated on an additional layer of dielectric (also $h=1.5 \mathrm{~mm}$, see left side on Fig. 2) in order to increase its bandwidth [11]. This approach provides higher efficiency, compared to the case where an additional layer of dielectric is placed over the whole structure. Port 1 is connected directly to the CPW, exciting the even mode and producing LHCP radiation. A microstrip line on the top side of the substrate (connected to port 2) couples the odd mode signal to the CPW (similarly to [9]) and produces RHCP. Since the microstrip passing over CPW introduces unwanted reactance for the even mode, it is advantageous to make the microstrip width $W_{m}$ as narrow as possible. On the other hand the microstrip needs to be matched to $50 \Omega$. As a compromise, a tapered impedance transformer was used, which allows a decrease of $W_{m}$ without mismatch. The lengths $L_{m 1}$ and $L_{m 2}$ were initially set so that $L_{m 1}+L_{m 2}$ is approximately a quarter wavelength and were later optimized by a CST parameter sweep. For the second port the odd mode is filtered out by an SMA connector, as its outer shell connects the two most outer conductors of CPW (alternatively a bridge can be implemented, similarly to [12]). From the CPW line (inner conductor: $2.4 \mathrm{~mm}$ wide, slots: $0.2 \mathrm{~mm}$ ) the two slots are separated. A phase shifter is realized by prolonging one of the slots [12] by a distance of $\Delta L_{s}=2\left(L_{s 1}-L_{s 2}\right)$. To enhance symmetry of the patch feedpoint amplitudes, the shorter slotline is designed to incorporate one bend more than the longer slotline with phase shifter. This symmetry improves the AR of both RHCP and
LHCP modes. Finally the energy is coupled into the patch, located on the elevated substrate. To ensure a good match the slots are triangularly extended, so that $W_{t}>h$.

The dimensions of the structure are as follows: $W_{s}=85 \mathrm{~mm}, L_{s}=80 \mathrm{~mm}, W_{p}=L_{p}=31 \mathrm{~mm}, L_{t}=6 \mathrm{~mm}$, $W_{t}=4.4 \mathrm{~mm}, \Delta_{t}=1.3 \mathrm{~mm}, L_{q}=12.7 \mathrm{~mm}, L_{s 1}=13.5 \mathrm{~mm}, L_{\mathrm{s} 2}$ $=3 \mathrm{~mm}, L_{m 1}=11.3 \mathrm{~mm}, L_{m 2}=13.7 \mathrm{~mm}, W_{m}=1.7 \mathrm{~mm}$, $\theta_{m}=70^{\circ}$.

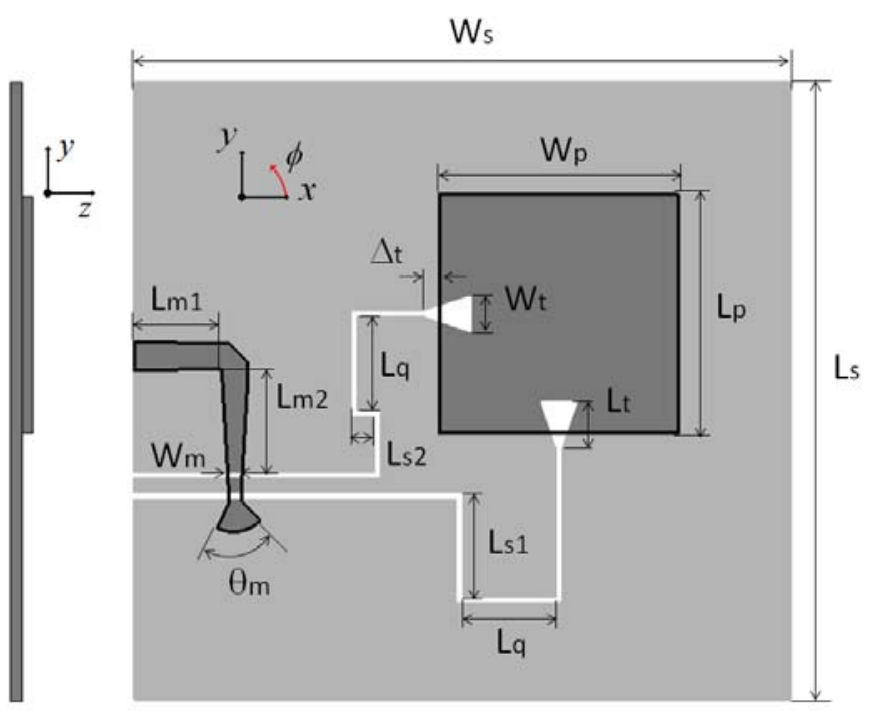

Fig 2. Proposed design with key dimensions. Black line represent edges of the metalization on top layer, light grey color - ground plane, white color - slots in ground plane

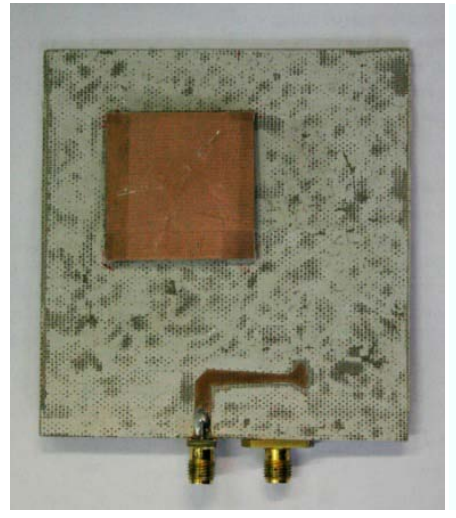

a)

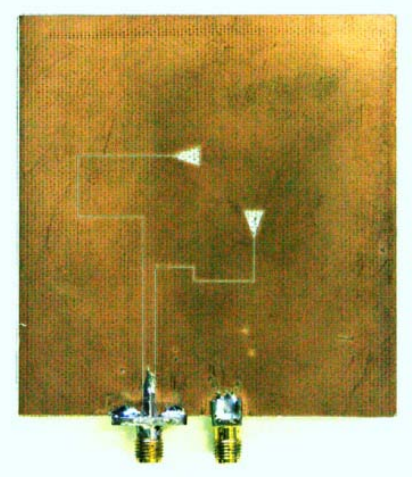

b)
Fig 3. Photographs of the proposed antenna.(a) front side; (b) rear side

\section{RESULTS AND DISCUSSION}

The structure was simulated using the CST Microwave Studio time-domain solver. Fig. 4 shows the reflection coefficients and isolation between the two input ports. As the device is reciprocal, $\left(S_{12}=S_{21}\right)$ only one curve is shown for clarity. It can be seen that the measured port-to-port isolation is up to $20 \mathrm{~dB}$ at the patch resonant frequency and is better than $10 \mathrm{~dB}$ across the band of $2.515-2.598 \mathrm{GHz}$ (3.2 \%).

The coupling mechanism between microstrip and CPW introduces some phase and amplitude error between the two slots and the optimum axial ratio (AR) is at a slightly higher 
frequency for the odd mode than for the even one. Despite this, there is a good CP and impedance performance (all Sparameters are below $-10 \mathrm{~dB}$ ) for both modes in the band $2.557-2.591 \mathrm{GHz}$, giving a 3dB AR bandwidth of $34 \mathrm{MHz}$ (1.3\%), which is acceptable for a microstrip patch antenna.

It can be seen in Figs. 6-9, that the proposed antenna provides two orthogonal $\mathrm{CP}$ modes, depending on the feed port. The boresight axial ratio at $2.575 \mathrm{GHz}$ is $2.45 \mathrm{~dB}$ for port 1 and $1.82 \mathrm{~dB}$ for port 2 . The measured realized gains are 7.4dBic for LHCP in port 1 and $4.8 \mathrm{dBic}$ for RHCP in port 2. The difference is most likely due to the loss in the coupling mechanism. The additional length of the phase shifter impacts both RHCP and LHCP simultaneously and hence does not contribute to this difference.

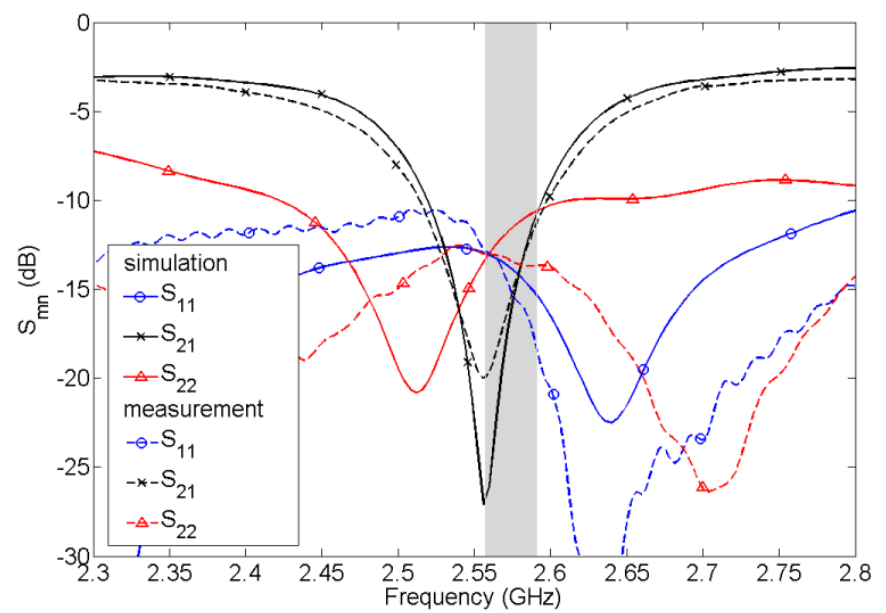

Fig 4. Reflection coefficients and port-to-port isolation. Grey area represents frequency range with $\mathrm{AR}<3 \mathrm{~dB}$ for both ports.

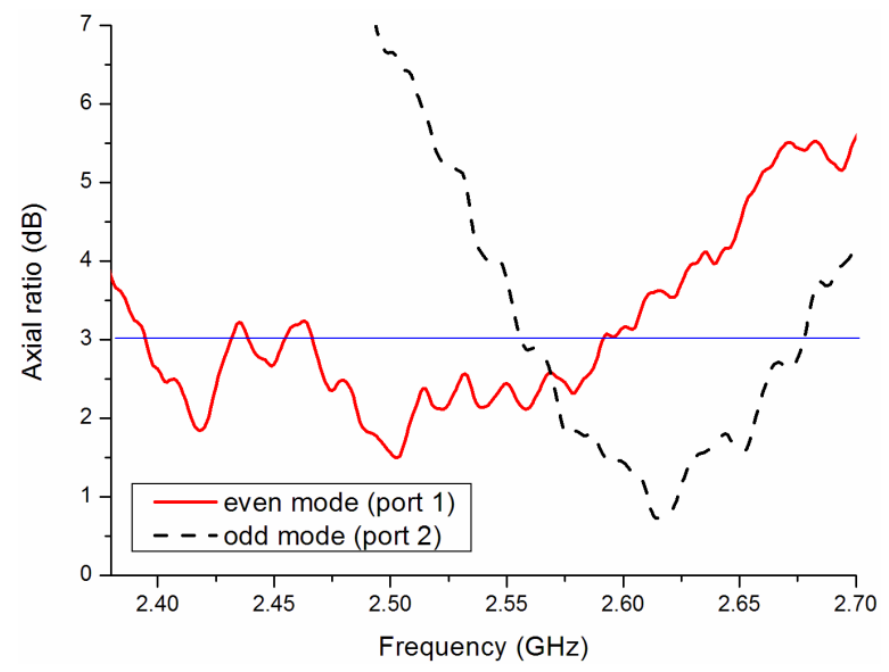

Fig 5. Measured AR at boresight as a function of frequency.

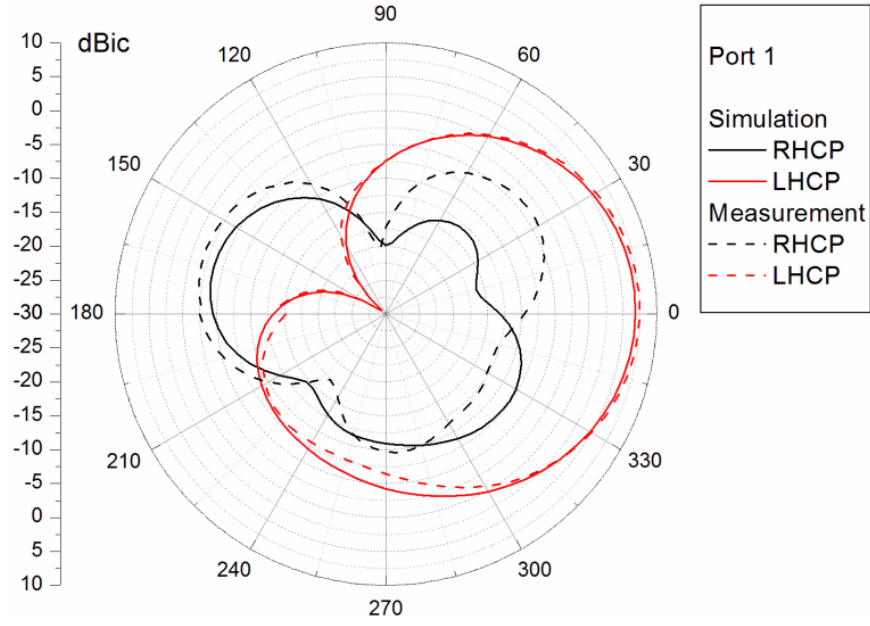

Fig 6. CP realized gain in $y z$ plane for port 1 (even mode)

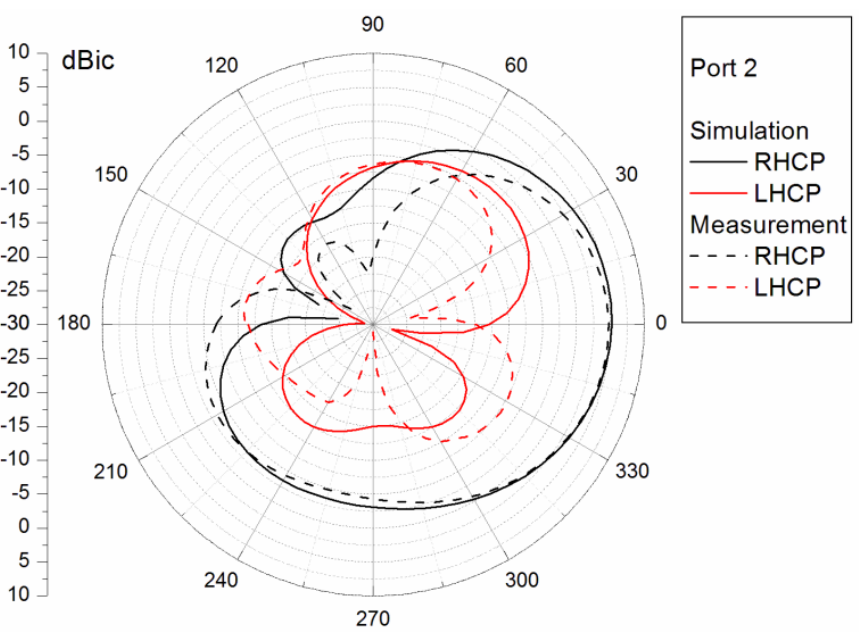

Fig 7. CP realized gain in $y z$ for port 2 (odd mode)

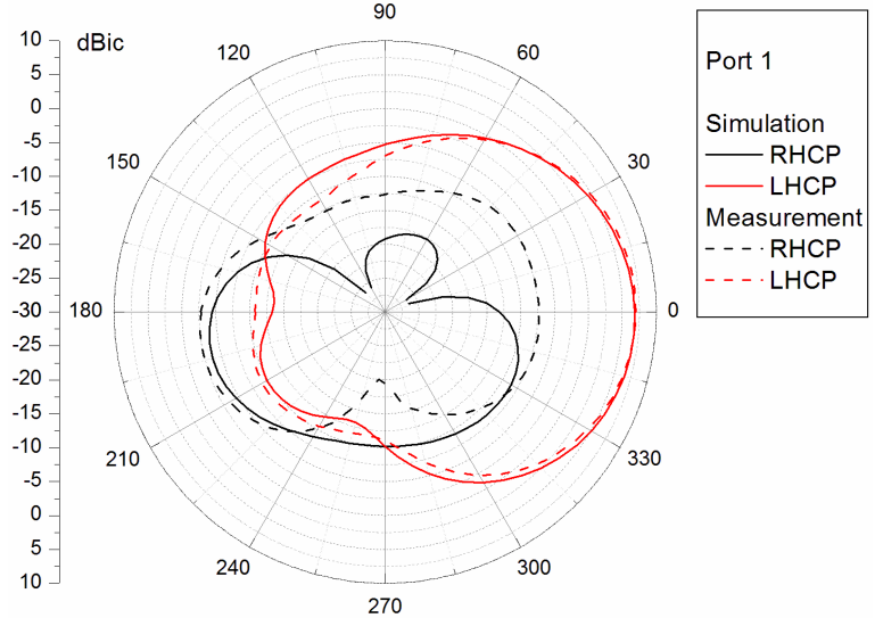

Fig 8. CP realized gain in $x z$ for port 1 (even mode) 


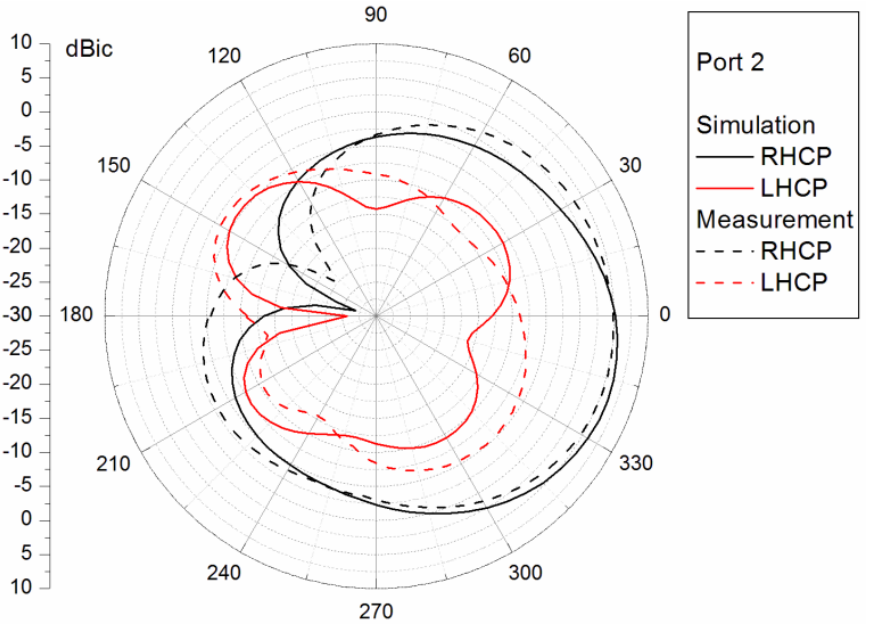

Fig 9. CP realized gain in $x z$ for port 2 (odd mode)

\section{ISOLATION}

Isolation between RHCP and LHCP ports is a crucial issue for potential applications. However, due to the use of ${ }^{\circ} 90$ phase shifter, the principles for achieving good and wideband isolation are different to those presented in [9]. Fig. 10 illustrates the phase of the signal, which is reflected from the patch. The odd mode is excited, hence 0 phase difference at the input. As the wave travels through the line a $90^{\circ}$ phase difference is added by the phase shifter. When the energy is reflected from the patch (red dashed arrows) the signal travels back and through the phase shifter for the second time, effectively producing $180^{\circ}$ phase shift. As a result - although excitation is in odd mode - the reflected wave is in even mode (and vice versa for the even mode excitation). This shows that the limiting factor for good isolation is the patch impedance matching and its bandwidth. This is especially critical because traditional patch antennas are narrowband. The use of bandwidth enhancement techniques ([11], [13]) is therefore strongly recommended for the proposed designs.

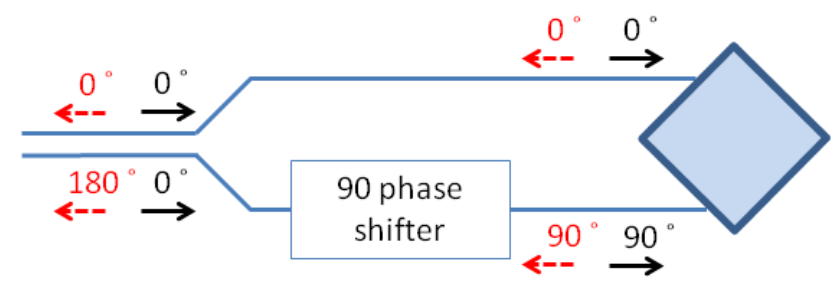

\section{$\rightarrow$ Forward signal (odd mode excitation) «-- Signal reflected from the patch}

Fig 10. The phase differences for the forward and reflected signals for odd mode excitation. For even mode excitation the directions of the arrows are reversed.

\section{CONCLUSIONS}

In this communication a new antenna for circular polarization diversity is proposed. Good isolation in the operating band makes the antenna a suitable candidate for wireless applications. Other applications include polarization sensor antennas. Apart from the $90^{\circ}$ phase shifter (which can be easily realized within a limited footprint) the antenna feed has no wavelength dependent elements, therefore providing a compact solution. The use of a single CPW to transmit two separated signals allows a flexible design, especially in small devices where the space available for RF circuits is constrained.

The use of CPW and a coupling mechanism to generate the odd mode was used to facilitate measurement with a VNA. In fact almost any line supporting even/odd modes can be employed and the two modes can be generated in various ways, for instance directly by a MMIC, as even and odd modes are often used in microwave circuits.

\section{REFERENCES}

[1] P. R. King, T. W. C. Brown, A. Kyrgiazos, and B. G. Evans, "Empiricalstochastic LMS-MIMO channel model implementation and validation," IEEE Trans. on Antennas and Propagation, vol. 60 (2), pp. 606-614, Feb. 2012.

[2] F. Yang, and Y. Rahmat-Samii, "A reconfigurable patch antenna using switchable slots for circular polarization diversity," IEEE Microwave and Wireless Components Letters, vol. 12 (3), pp. 96-98, March 2002.

[3] R.-H. Chen,and J.-S. Row, "Single-fed microstrip patch antenna with switchable polarization," IEEE Trans. on Antennas and Propagation,vol. 56 (4), pp. 922-926, Apr. 2008.

[4] B. Wu, and M. Okoniewski, "A novel scheme for realizing a microstrip antenna with switchable circular polarization," in Proc. EuCAP'12 - 6th European Conference on Antennas and Propagation, Prague, Czech Rep., 2012, pp. 3278-3282.

[5] B. A. Centiner, J. Y. Qian, G. P. Li, and F. De Flaviis, "A reconfigurable spiral antenna for adaptive MIMO systems," EURASIP Journal on Wireless Communication and Networking, issue 3, pp. 382-389, Aug. 2005.

[6] J.-S. Row,and C.-J. Shih "Polarization-diversity ring slot antenna with frequency agility," IEEE Trans. on Antennas and Propagation, vol. 60 (8), pp. 3953-3957, Aug. 2012.

[7] F. Ferrero, C. Luxey, R. Staraj, G. Jacquemod, M. Yedlin, and V. Fusco, "A novel quad-polarization agile patch antenna," IEEE Trans. on Antennas and Propagation, vol. 57 (5), pp. 1563-1567, May 2009.

[8] F. Ferrero, C. Luxey, R. Staraj, G. Jacquemod, M. Yedlin, and V. Fusco, "Theory and design of a tunable quasi-lumped quadrature coupler," Microwave and Optical Technology Letters, vol. 51 (9), pp. 2219-2222, Sep. 2009.

[9] Y. Li, Z. Zhang, W. Chen, Z. Feng, and M. F. Iskander, "A DualPolarization slot antenna using a compact CPW feeding structure," IEEE Antennas and Wireless Propagation Letters, vol. 9, pp. 191-194, Mar. 2010.

[10] K. C. Gupta et al., Microstrip Lines and Slotlines. Belmont, CA: Artech House, 1996, pp. 269-291.

[11] W.L.Langston, and D.R.Jackson, "Impedance, axial-ratio, and receivepower bandwidths of microstrip antennas," IEEE Trans. on Antennas and Propagation, vol. 52 (10), pp. 2769-2774, Oct. 2004.

[12] E. A. Soliman, S. Brebels, E. Beyne, and G. A. E. Vandenbosch, "Circularly polarised aperture antenna fed by CPW and built in MCM-D technology,” Electronic Letters, vol. 35 (4), pp. 250-251, Feb. 1999.

[13] N. Herscovici, Z. Sipius, and D. Bonefacic, "Circularly polarized singlefed wide-band microstrip patch," IEEE Trans. on Antennas and Propagation, vol. 51 (6), pp. 1277-1280, Jun. 2003. 